\title{
Coulisses
}

Revue de théâtre

25 | Hiver 2002

Varia

\section{L'ANRAT en région Franche-Comté}

\section{Jean-Christophe Delbende}

\section{OpenEdition}

Journals

Édition électronique

URL : http://journals.openedition.org/coulisses/6024

DOI : $10.4000 /$ coulisses. 6024

ISSN : 2546-9460

\section{Éditeur}

Presses universitaires de Franche-Comté

\section{Édition imprimée}

Date de publication : 1 janvier 2002

Pagination : 102-103

ISBN : 2-84627-052-X

ISSN : $1150-594 \mathrm{X}$

\section{Référence électronique}

Jean-Christophe Delbende, «L'ANRAT en région Franche-Comté », Coulisses [En ligne], 25 | Hiver 2002, mis en ligne le 24 octobre 2019, consulté le 15 novembre 2019. URL : http://journals.openedition.org/ coulisses/6024; DOI : 10.4000/coulisses.6024

Ce document a été généré automatiquement le 15 novembre 2019.

Coulisses 


\title{
L'ANRAT en région Franche-Comté
}

\author{
Jean-Christophe Delbende
}

1 L'Association nationale de recherche et d'action théâtrale en milieu scolaire et universitaire est un mouvement indépendant dont la finalité est de favoriser le développement des pratiques artistiques du théâtre à l'école et de faciliter le dialogue entre artistes, structures culturelles et enseignants.

\section{Des rencontres bourguignonnes aux franc-comtoises}

2 C'est à l'issue des Rencontres régionales Théâtre-Éducation de Bourgogne, au CDN de Dijon, en janvier 2001 que, quelques militants ${ }^{1}$ de l'ANRAT et moi-même, avons eu la ferme conviction qu'une telle manifestation en Franche-Comté ne pourrait que servir notre engagement. Certes, chacun nourri de nos propres motivations: pour l'un, raviver une dynamique locale théâtre/éducation riche dans les années 80, pour l'autre, permettre que la réalisation de projets théâtre à l'école ne relève plus du parcours du combattant, pour moi enfin, trouver une aide à la réalisation du plan départemental théâtre que je développe dans le cadre de ma mission Maîtrise des langages dans le $1^{\mathrm{er}}$ degré.

\section{Des réunions préparatoires à la constitution d'un groupe de pilotage}

3 Au regret de constater que la majorité des personnes présentes était différente d'une réunion à l'autre, s'est substituée l'assurance d'obtenir une très large représentativité des sphères que nous voulions mettre en contact!

\section{Le 29 septembre à l'Espace}

Un hôte ${ }^{2}$, des partenaires ${ }^{3}$, des participants (150), et bien sûr, l'ANRAT ${ }^{4}$, (de moins en moins parisienne à force de régionales!), des thèmes de réflexion articulés autour de 
trois ateliers : la formation, le rôle des collectivités locales et territoriales, le projet culturel, mais... pas de grand-messe : des rencontres! Et si les Actes ${ }^{5}$ sont à gestation longue (parution en janvier 2002), le groupe ANRAT en région Franche-Comté, l'une des finalités de cette manifestation, s'est déjà constitué et réuni, chez un nouvel hôte, le Nouveau Théâtre, le $1^{\text {er }}$ décembre 2001.

\section{L'ANRAT en région... Franche-Comté}

C'est un lieu neutre d'échange et de rencontre qui définit ses champs de réflexion propre en fonction de son actualité. L'ANRAT n'étant pas une fédération d'associations, le collectif régional ne s'érige donc pas en association indépendante; c'est avant tout en tant que militants de l'ANRAT que les membres du groupe se réunissent.

L'équipe s'est fixé comme modalité de fonctionnement d'alterner des réunions de travail en groupe restreint (réservées aux adhérents de l'ANRAT) et des réunions d'information ouvertes à tous.

Notre premier rendez-vous, large public, le $1^{\text {er }}$ décembre au CDN, a permis de faire une mise au point sur les dispositifs financés par l'Éducation nationale et/ou DRAC: AEI (action éducative innovante), AA (atelier artistique), PAC (projet artistique et culturel), API (actions pédagogiques innovantes, $2^{\mathrm{e}}$ degré) : spécificité de chaque dispositif, dans son mode de financement, sa "philosophie », ses aspects pédagogiques et artistiques. Le dispositif PAC, encore peu connu, appelle de nombreuses questions qui traduisent une inquiétude des compagnies : ces dernières doivent-elles être " parrainées » par une structure culturelle pour intervenir dans les écoles? Si les textes n'imposent pas ce "parrainage », une forte incitation dans ce sens est préconisée par le Plan pour les arts et la culture, co-signé par les ministères de l'Éducation nationale et de la Culture. Il ne s'agit pas de systématiser une démarche, mais d'essayer de faire coïncider la création de l'artiste avec le projet pédagogique de la classe. Il s'agit là d'une exigence, inscrite dans les textes. Et la force du partenariat enseignant artiste (ou compagnie) et structure culturelle peut servir cette exigence. Ce débat, loin d'être clos, investira nécessairement le thème de nos prochaines réunions : la formation conjointe.

\section{La prochaine réunion élargie}

C'est-à-dire ouverte à toute personne intéressée par la problématique théâtreéducation et qui souhaite avancer avec l'ANRAT, aura lieu samedi 9 mars 2002, de 15 heures à 22/23 heures (avec pause repas) au Théâtre Edwige Feuillère de Vesoul (ThéV, 66 place Pierre-Renet, 70000 Vesoul). Cet horaire est choisi afin de permettre aux enseignants qui travaillent le samedi matin de participer sans demande de dérogations. Les inscriptions se feront directement auprès du ThéV (tel. $03847540 ; 66$, courriel vesoul.theatre@ac-vesoul.fr).

Le thème de la formation conjointe enseignants-artistes fera l'objet de tables rondes (quand? pourquoi ? comment? dans ou hors institution? complémentarité des deux pôles enseignant artiste, définition d'un type de partenariat pour trouver un type de formation.), mais aussi d'ateliers (échange des pratiques). 


\section{Quant à l'équipe permanente de l'ANRAT (3 permanents)}

7 Elle s'appuie sur les structures (rectorats, inspections académiques, DRAC, théâtres, compagnies, etc.), tout en restant une association indépendante dont la politique est fixée par le conseil d'administration et les adhérents.

Elle demande aux DRAC et aux rectorats d'attribuer des moyens à des coordinateurs/ animateurs du réseau régional. Exemple de Dijon où la DRAC a donné des moyens à deux structures et le rectorat déchargé à hauteur de deux fois 5 heures des enseignants pour assurer les tâches de coordination au sein des deux structures culturelles de référence. Exemple encore, à Angers, où cette situation existe de fait depuis de nombreuses années avec une grande réussite. En attendant l'attribution de moyens humains et financiers, l'association prend en charge les frais d'organisation logistique sur facture dans la limite des possibilités.

Elle est disponible pour aider à avancer, discuter des ordres du jour, venir à certaines réunions donner des informations. Les décisions d'actions importantes sont prises en lien avec l'équipe permanente de l'ANRAT et son conseil d'administration. Exemple, le $1^{\text {er }}$ décembre, Céline Teurquéty, chargée de communication de l'ANRAT, s'était déplacée à Besançon pour faire le point sur les rencontres régionales à venir (Bretagne, Haute-Normandie, Midi-Pyrénées, Languedoc-Roussillon, Aquitaine, Région PACA) et sur les modalités de travail d'autres groupes régionaux (Ile-de-France, Nord).

Elle met à disposition un espace dans ses publications pour diffuser le fruit des recherches régionales.

\section{NOTES}

1. Jacques Vingler, Yves Courty, Jean Martin.

2. Et néanmoins partenaire : la Scène nationale de l'Espace, Daniel Boucon et toute son équipe.

3. Rectorat, DRAC, inspection académique, CRDP, CDDP, conseil général, Ville de Besançon.

4. En la personne de son délégué national, Jean-Pierre Loriol.

5. Les personnes n'ayant pas participé aux rencontres du 29/09/01 et souhaitant recevoir les actes peuvent contacter Jean-Christophe Delbende au 0381654850 .

\section{AUTEUR}

JEAN-CHRISTOPHE DELBENDE

ANRAT Franche-Comté 